\title{
The Hotspots and Trends of Adaptive Learning: A Visualized Analysis Based on CiteSpace
}

\author{
Miaomiao Zhang and Rui Zhang
}

\begin{abstract}
Using the visual analysis of CiteSpace, and adopting the visual analysis and content analysis as main research methods, research on hot spots and development trends of adaptive learning were revealed. The literatures were collected from 2000 to 2018 . The study of adaptive learning technology in foreign countries focus on higher education, and it is suggested to expand the scope of the research. The application of adaptive learning technology in education should be strengthened in China. The updating and integration of learning style scale should be considered and the open learner model is a new trend in future. In order to achieve the sharing of resources and domain models or the reusability and portability of the system, unified standards should be established. In addition, we should pay attention to the application of learning science theory closely related to adaptive learning. Adaptive personalized support service with big data, artificial intelligence, context recognition and emotion capture are a new trend in this field.
\end{abstract}

Index Terms-Adaptive learning, CiteSpace, co-word analysis, visual analysis.

\section{INTRODUCTION}

With the advent of mobile learning, ubiquitous learning, lifelong learning, and the era of education big data, adaptive learning is receiving more and more attention from the education community. A survey conducted by the United States Higher Education Internal Reference and Gallup institutions shows that $66 \%$ of respondents believe that adaptive learning has a greater positive impact on higher education than MOOC [1]. Adaptive learning technology can realize individualized learning support services by dynamically establishing learner models, learning path recommendations, and data evaluation, and improve learner learning performance. This paper first provides an overview of adaptive learning in the literature, then predicts the future development trend, and finally gives reasonable suggestions based on the current research status and application of adaptive learning in China.

\section{DATA SOURCES AND RESEARCH METHOdS}

\section{A. Data Sources}

Manuscript received June 19, 2019; revised February 29, 2020

Miaomiao Zhang is with the School of Education Information Technology, Capital Normal University, Wuhan, China (e-mail: 599153085@qq.com).

Rui Zhang is with the Educational Information Technology Collaborative Innovation, Center Capital Normal University, Wuhan, China (e-mail 1837693345@qq.com).
This article uses Web of Science database as the data source, and the subject of "Adaptive Learning", "Adaptive e-learning systems", "Intelligent Tutoring Systems", "Adaptive Educational Hypermedia", "Adaptive Hypermedia System" are used for accurate retrieval. There's no time limit. The paper selected 10 kinds of educational technology SSCI journals such as "Computers \& Education", "British Journal of Educational Technology" and "Journal of Computer Assisted Learning". There are 451 articles after the irrelevant articles are removed. The selection criteria of the articles are: articles that are highly relevant to adaptive learning, adaptive, and adaptive learning systems; excluding irrelevant articles such as children and middle school students' learning adaptability; conference papers and reviews are not included.

\section{B. Research Methods}

In this paper, the visual analysis method and content analysis method are used, and then the CiteSpace knowledge visualization tool [2] is used to analyze the keyword and keyword co-occurrence network for the related topics of "adaptive learning". In order to gain a deeper understanding of the detailed information behind the CiteSpace map, I will perform a second search of the literature based on the map information, and then use the content analysis method to carefully read the articles in the map, including the turning points, marking points, height centers, and hot topics, and systematically combing and critically reading the relevant documents

\section{ADAPTIVE LEARNING RESEARCH HOTSPOTS}

Key words co-occurrence analysis is used to predict the research hotspots and development trends of a certain period of time by establishing a co-occurrence matrix between keywords and keywords.

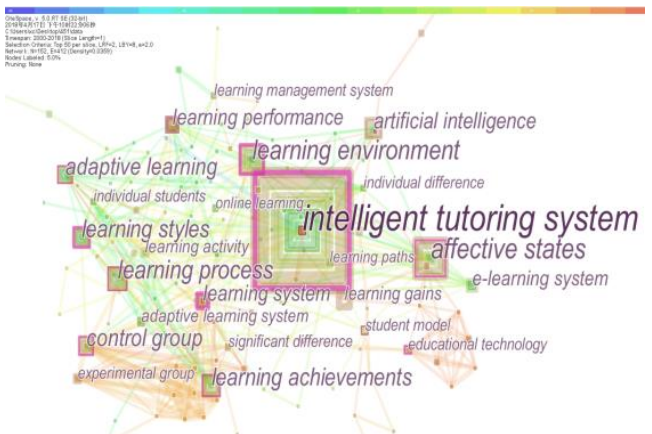

Fig. 1. Keyword analysis map.

The 451 articles obtained from the Web of Science database were used as data sources. The time range was set from 2000 to 2018 , and the time slice was set to 1 year. The 
burst value list reflected the high degree of attention of the emerging keywords in a certain period, indicating that some of the research content is the research focus and possible potential value in the corresponding period. Similarly, the subject analysis graph and the burst value are shown in "Fig. 1 " and "Fig. 2":

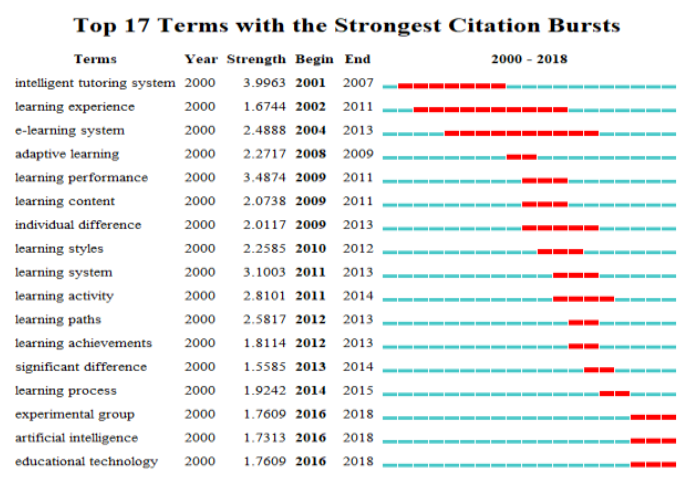

Fig. 2.The top seven keywords of the burst value.

See Table I for the top 12 keywords based on the frequency of citations:

TABLE I: LIST OF HIGH FREQUENCY SUB-HEADINGS
\begin{tabular}{|c|c|c|}
\hline Citation counts & Centrality & References \\
\hline 74 & 0.43 & intelligent tutoring system \\
\hline 26 & 0.19 & affective states \\
\hline 23 & 0.29 & learning environment \\
\hline 18 & 0.13 & learning progress \\
\hline 17 & 0.2 & learning style \\
\hline 17 & 0.18 & learning achivements \\
\hline 14 & 0.16 & control group \\
\hline 14 & 0.12 & adaptive learning \\
\hline 13 & 0.07 & artificial intelligence \\
\hline 12 & 0.42 & learning system \\
\hline 12 & 0.15 & learning performance \\
\hline 6 & 0.17 & student model \\
\hline
\end{tabular}

Combined with "Fig. 1" and "Table I", it can be seen that the main themes of adaptive learning research include intelligent tutor system, learning emotional state, learning environment, learning style, learner model, artificial intelligence, learning system, and learning performance. This paper divides the foreign adaptive learning research hotspot into three stages according to the co-occurrence analysis of the topic words and the terms with strongest citation bursts:

\section{A. Research Hotspots in the Rise Phase: 1996-2008}

The main keywords are intelligent tutors and adaptive education hypermedia. The adaptive learning articles in this stage focus on the research of adaptive education hypermedia, which is based on human-computer interaction, machine learning, cognitive science, and constructivist learning theory, such as Bloom's taxonomy, cognitive load theory, knowledge space theory, learning style, control-value theory, cognitive evaluation theory of emotion, multi-attribute decision making, the theory of Human Plausible Reasoning (HPR),
Carol model, item response theory. Research at this stage focuses on developing systems, building frameworks, and building models.

The modeling methods of student models in the literature generally use eight methods, namely superposition model, type model, perturbation model, cognitive modeling, machine learning, fuzzy calculation, ontology and bayesian. Modeling is mainly from the perspective of learning style, cognitive ability, knowledge level, and learning objectives. The literature is modeled from the perspectives of learning style, cognitive ability, knowledge level, and learning objectives. A large number of AEH studies focus on user model modeling based on learning style. There are two methods of learning style measurement or automatic modeling. The learning style scale uses more Felder and Silverman, Kolb, Honey and Mumford scales. A study based on kolb's ELT empirical learning theory incorporates learning style into adaptive e-learning hypermedia to develop a new adaptive online hypermedia system [3]. However, there are two problems with the model based on the learning style scale: First, the questionnaire is long, boring, and time consuming [4]; second, the learner does not know his own learning style and learning preferences. And Hwang G J investigated whether students have the ability to choose an adaptive learning system that fits their learning style, and the results show that many students rely on intuitive choices [5].Therefore automatic modeling can produce a better student model.

\section{B. Research Hotspots at the Development Stage: 2008-2012}

Research hotspots include system development, model modification, verifying system effectiveness. And keywords include adaptive learning, learning performance, learning content, individual differences, learning paths, and academic performance. Professor Peter Brusilovsky of Cameron University proposed the open student modeling (OSM), which allows learners to explore their own learning models, and then proposes an open social student model which allows learners to explore peers or learners in the same cluster. The function of the model complements the lack of OSM in social cognition [6].

Table I shows the frequency and centrality of academic achievement, learning performance, and control group as keywords. It shows that there are many researches on the effectiveness of adaptive learning systems, including the effectiveness detection of automatic modeling and the effectiveness of the system. The results are mainly verified from the perspectives of learner satisfaction, system friendliness, learner achievement, and learning experience. The methods used in the literature are generally empirical studies and questionnaires. A study has used PeRSIVA to evaluate the effectiveness of student modeling and diagnosis from the perspective of student satisfaction, performance, progress, behavior, and status [7]. Hwang G J developed an adaptive learning system based on the two main sources of learning styles and learning behaviors, and tested the effectiveness of the system. Experiments show that the system can improve students' academic performance and learning efficiency [8]. In addition, he developed an adaptive 
learning system from the perspective of dividing learning materials into different learning objects according to curriculum standards [9]. A study used questionnaires to collect student satisfaction and then used spatial density-based clustering algorithms for analysis, and the study helped to improve the adaptive learning support service by solving the problem that the respondent did not complete the questionnaire or did not express real ideas. [10].

\section{Research Hotspots in the Emerging Stage: 2012-2018}

Research hotspots include adaptability, ontology, semantic web, data mining, and machine learning technologies in the mobile environment; most of the research on adaptability in the mobile environment is concentrated in 2012 to 2017: First from the perspective of mobile learning, the time and place of student learning is constantly changing. And contextual awareness plays an important role in learning. Research has combined vocabulary and machine learning techniques to develop a Facebook application. It retrieves information written by the user, extracts information about the user's emotional state, and classify emotions into positive, neutral, and negative categories. Its accuracy rate of emotional status is over $83 \%$, which helps to understand the potential needs of learners at the time. [11]. A study introduces an adaptive mobile system in the mobile environment that takes into account the learner's environment and the learner's unique learning style, using distance sensors, device sensors, GPS sensors, context models, user databases, and adaptive engines to determine the best presentation of the course [12]. Second, the seamless connection between online learning and mobile learning. The adaptive learning system needs to have a device attribute detection mechanism. It can adjust content, display form, and function according to the format and attributes of different devices. A study has proposed an adaptive mobile learning system that uses bayesian network and content adaptation technology to support learner adaptation and device adaptation [13]. Research has developed a cloud-based adaptive learning system that enables seamless connectivity between online learning and mobile learning [14]. A study has developed an adaptive learning system based on mobile email. The system calculates the frequency of each word in different types of news by searching the RSSs of the Japanese news website to find the words commonly used in the field, and then push the words and sentences of the corresponding fields to the learners [15].

The research of data mining and fuzzy logic technology applied in adaptive learning system has gradually increased since 2008. Data mining analyzes the learning behavior patterns of learners by analyzing the large amount of data generated during the student learning process, which is beneficial to personalized learning path recommendation. Fuzzy logic helps to solve the uncertainty problem in learning by deducting the thinking of the human brain. Some research use data mining technology to cluster learners according to learning styles, and divide learners into different groups. Experiments show that the adaptive courses improve student achievement [16]. A study has developed a self-adaptive personalized learning system based on fuzzy logic. It uses fuzzy reasoning to find articles that are appropriate for the learner's level of difficulty, and then builds applicability formulas. This method not only improves the learner's English ability, but also helps to maintain the learner's interest in learning [17].

\section{FUTURE DEVELOPMENT TRENDS}

Technology continues to innovate and learning styles are constantly changing. As mentioned above, adaptability in the mobile learning environment is also the research trend of adaptive learning. The comprehensive application of semantic web, data mining, machine learning technology and ontology technology is conducive to the realization of knowledge sharing, the establishment of rich semantic models, tracking and learning learner learning paths, mining large amounts of data, and accurate diagnosis. It will be the mainstream direction of future research. The uncertainties of fuzzy computing, bayesian methods for processing, diagnosing models and the modeling of learner emotional states are also increasingly concerned by researchers.

\section{A. Personalized Adaptive Support Services}

Personalized learning is increasingly valued by the government and education. The adaptive learning system provides suggestions for learners through continuous interaction. Rich and personalized learning resources, timely feedback systems, fast communication systems, and adaptive real-time assessments are more in line with individual needs. The arrival of the era of education big data is a good opportunity to achieve personalized learning. The breakthrough required for the future MOOC to achieve large-scale and personalized integration is to enhance the content optimization design and intelligence of adaptive terminals.

Personalized adaptive learning will become the new normal in the era of education big data. At present, domestic personalized adaptive learning is still in the stage of theoretical research and small-scale exploration. In the future, personalized adaptive learning uses data mining for learning and analysis, which is beneficial to learners' personalized learning. MOOC has the characteristics of large users and abundant resources, but it lacks in adaptive learning. A study uses quantitative self-algorithms to establish an educational big data of adaptive learning MOOC system model centered on learners' cognitive needs. Big data supports learning process behavior records, making learning more transparent, supporting data visualization, and facilitating learning management. The combination of big data and adaptive systems can realize learner's personalized learning. In future personalized adaptive learning will become a new paradigm for educational technology research in education big data.

\section{B. Context-Aware Adaptive Technology}

With the development of ubiquitous learning and mobile learning, adaptive technology should pay more and more attention to the context of learners and the learning emotional state of learners. Adaptive technology based on emotional state and context perception is the development trend of adaptive learning technology in the future.

The premise of the adaptive learning system is that the learner is always in the learning state, but in fact the learner 
may face various difficulties and have anxiety, boredom and other emotions. Therefore the adaptive learning support service should also pay attention to and capture the learner's learning state in time. It should adopt certain strategies to encourage learners to keep learning. However, adaptive learning still needs to be broken in emotional support. Face recognition, skin sensing, location perception and environment perception are used to determine the learner's current learning state. Real-time monitoring and management of learners' learning emotions are also the direction of future research.

\section{Adaptive Education Based on Artificial Intelligence}

Foreign artificial intelligence is mainly for learner's adaptive learning, and China is still in its infancy. The application of artificial intelligence to adaptive learning is mainly reflected in question answering, learning emotion capture, learning resources, intelligent push, fast positioning learning methods and learning content. Artificial intelligence has functions such as intelligent cognitive algorithms, pattern recognition, and intelligent sensing technology. The adaptive education model supported by artificial intelligence technology can achieve a virtuous circle of domain models and teaching models and enhance learners' learning experience.

\section{CONCLUSIONS AND PROSPECTS}

1) Foreign adaptive learning research is more extensive and mature, but practical research focuses on higher education, and it is recommended to expand the scope of research in the future. The domestic adaptive learning research focuses on theoretical research, and there are few studies on educational practice.

In China, Yifei Li and Xinming Zhu first proposed human adaptive learning. They elaborated on the theory and practice of adaptive learning in 1998 and 2004.The basic theoretical research of adaptive learning includes the concept, characteristics, classification and theoretical basis of adaptive learning. Shengquan Yu proposed the concept of adaptive learning in 2000. He think that adaptive learning is a resource-based learning. It is the active learning of learners. Learners actively construct knowledge in an adaptive environment. The core features are personalized, individualized, timely feedback from teachers or peers. There are few studies on learning strategies, teaching strategies, learning theories, and teaching theories to guide adaptive learning. The application research of learning science and pedagogy theoretical achivements in adaptive learning should be further explored. In 2011, Qiang Jiang and Wei Zhao proposed the design specifications of domain models and user models in adaptive learning systems. They proposed an empirical study to correct the learning style model through data mining of students' online learning behavior. In addition, Qiang Jiang and Wei Zhao pointed out that the filling of the learning style scale may result in inaccurate test results due to staticity, random filling of learners, and strong subjective judgment. It is proposed to use bayesian network to explore learning behaviors and dynamically establish a learning style model.
The applications of domestic adaptive learning mainly include Zhixue.com and a series of English intelligent learning systems such as VIPKID and English Fluency. Zhixue.com realizes personalized and knowledge-based learning diagnosis through big data analysis. It can not only help students to find the root of the wrong questions, but also push matching micro-resources for students. In addition, it can also use smart terminal devices such as mobile phones, scanners, and scorers to realize programmatic teaching data collection under various teaching scenarios.

2) Research at home and abroad mostly uses the Felder and Silverman style scales for learner modeling.

The update and integration of the scale should be considered. The learner model is mostly a closed learner model in the system. With the development of MOOC and ubiquitous learning, the trend of the learner model is the open learner model. Thr open learner model allows learners to understand the learning state of themselves and peers. Open learner model is also conducive to the cultivation of learners' metacognition.

3) It is recommended to develop uniform industry standards to enhance system compatibility and portability and realize resources and knowledge base sharing.

The realization of high precision adaptation is not only the early coarse-grained knowledge, but also the subdivision of knowledge chapters and knowledge points. The mapping network between the knowledge map and the various knowledge chapters is formed, and the construction is highly relevant, portable, shared. Finally, a more complete knowledge domain model is formed.

By organizing the literature, I found that there is a lack of research among collaboration in the adaptive learning environment, the two-way adaptability of learners and systems, and the number of articles is small. From the perspective of learning theory, the sociality and negotiation of learning is a key factor in the knowledge construction, and we should pay attention to it. A study has combined the concepts of adaptive (system-controlled adaptation) and adaptive (user-controlled adaptation) to develop a negotiation-based adaptive learning system. When student preferences conflict with system recommendations, students negotiate with the system to reach an adaptation agreement [18]. There are also studies that integrate personalized adaptive online learning system(LECOMPS) with the honor-based social collaboration system(SOCIALX) to personalize learning paths and social collaboration functions [19]. In addition, the corresponding teaching mode of adaptive learning also conflicts with the existing teaching mode. Although learners are in an open adaptive learning system, most learners learn linearly according to the outline. And the real adaptive learning requires a revolution in culture and learning methods.

\section{CONFLICT OF INTEREST}

The authors declare no conflict of interest.

\section{AUTHOR CONTRIBUTIONS}

Miaomiao Zhang conducted the research and performed 
the data analyses and wrote the manuscript; Rui Zhang helped perform the analysis with constructive discussions; all authors had approved the final version.

\section{REFERENCES}

[1] A. Newman, P. Stokes, and G. Bryant. (April 2013). Learning to adapt: A case for accelerating adaptive learning in higher education. [Online]. Available:

http://tytonpartners.com/library/accelerating-adaptive-learning-in-high er-education

[2] C. Chen, CiteSpace: A Practical Guide for Mapping Scientific Literature, Nova Science Publishers, 2016.

[3] D. Samia and A. Abdelkrim, "An adaptive e-learning system based on students' learning styles: An empirical Study," International Journal of Distance Education Technologies, vol. 14, no. 3, pp. 34-51, July 2016.

[4] Y. Akbulut and C. S. Cardak, "Adaptive educational hypermedia accommodating learning styles: A content analysis of publications from 2000 to 2011," Computers \& Education, vol. 58, no. 2, pp. 835-842, February 2012.

[5] G. J. Hwang, H. Y. Sung, C. M. Hung, and I. Huang, "A learning style perspective to investigate the necessity of developing adaptive learning systems," Journal of Educational Technology \& Society, vol. 16, no. 2, pp. 188-197, April 2013.

[6] P. Brusilovsky, S. Somyürek, J. Guerra, and R. Hosseini,“' Open social student modeling for personalized learning," IEEE Transactions on Emerging Topics in Computing, vol. 4, no. 3, pp. 450-461, January 2016.

[7] K. Chrysafiadi and M. Virvou, "PeRSIVA: An empirical evaluation method of a student model of an intelligent e-learning environment for computer programming," Computers \& Education, vol. 68, no. 4, pp. 322-333, October 2013.

[8] J. C. R. Tseng, H. C. Chu, G. J. Hwang, C. C. Tsaic, "Development of an adaptive learning system with two sources of personalization information," Computers \& Education, vol. 51, no. 2, pp. 776-786,September 2008.

[9] S. Tseng, J. M. Su, G. J. Hwang, and G. H. Hwang, “An object-oriented course framework for developing adaptive learning systems," Journal of Educational Technology \& Society, vol. 11, no. 2, pp. 171-191, April 2008.

[10] A. Jeghal, L. Oughdir, H. Tairi, and A. Radouane, "Approach for using learner satisfaction to evaluate the learning adaptation policy," International Journal of Distance Education Technologies, vol. 14, no. 4, pp. 1,September 2016.

[11] A. Ortigosa, J. M. Martín, and R. M. Carro, "Sentiment analysis in facebook and its application to e-learning," Computers in Human Behavior, vol. 31, no. 1, pp. 527-541, February 2014.

[12] R. A. W. Tortorella and S. Graf, "Considering learning styles and context-awareness for mobile adaptive learning," Education \& Information Technologies, vol. 22, no. 1, pp.1-19, October 2015.
[13] H. C. Huang, T. Y. Wang, and F. M. Hsieh, "Constructing an adaptive mobile learning system for the support of personalized learning and device adaptation," Procedia - Social and Behavioral Sciences, vol. 64 no. 64, pp.332-341, November 2012.

[14] P. Nedungadi, "A new approach to personalization: integrating e-learning and m-learning," Educational Technology Research \& Development, vol. 60, no. 4, pp. 659-678, August 2012.

[15] H. Ogata et al., "Development of adaptive kanji learning system for mobile phone," International Journal of Distance Education Technologies, vol. 8, no. 4, pp.29-41, October 2010.

[16] A. Markovic et al., "Providing adaptivity in moodle LMS courses," Journal of Educational Technology \& Society, vol. 15, no. 1, pp 326-338, January 2012.

[17] T. C. Hsieh et al., "A fuzzy logic-based personalized learning system for supporting adaptive English learning," Journal of Educational Technology \& Society, vol. 15, no. 1, pp. 273-288, January 2012.

[18] C. Y. Chou et al., "Negotiation based adaptive learning sequences: Combining adaptivity and adaptability," Computers \& Education, vol. 88 , no. 1 , pp. $215-226$, October 2015

[19] M. D. Marsico et al., "A strategy to join adaptive and reputation-based social-collaborativee-learning," Through the Zone of Proximal Development, IGI Global, 2013.

Copyright (C) 2020 by the authors. This is an open access article distributed under the Creative Commons Attribution License which permits unrestricted use, distribution, and reproduction in any medium, provided the original work is properly cited (CC BY 4.0).

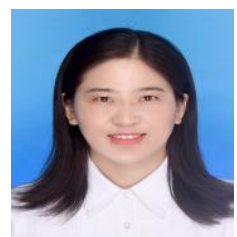

Miaomiao Zhang holds a B.S. in educational technology from Capital Normal University (China, 2012). After that, she worked at the School of Education Science, Nanyang Normal University. And she is now a postgraduate student at the School of Education Information Technology of Central China Normal University. Her major is education technology, and her research direction is the theory and practice of digital learning.

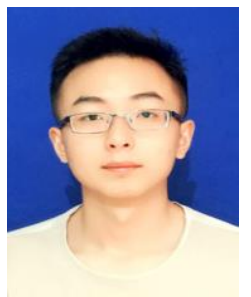

Rui Zhang is now a pastgraduate student at the Educational Information Technology Collaborative Innovation Center of Central China Normal University. His major is education technology, and his research direction is the theory and practice of learning. 